\title{
Ippolito II d'Este, les artistes et le royaume de France (1536-1563)
}

Ippolito II d'Este, gli artisti e il regno di Francia (1536-1563)

Ippolito II d'Este, Artists and the Kingdom of France (1536-1563)

\section{Jean Sénié}

\section{OpenEdition}

\section{Journals}

Édition électronique

URL : http://journals.openedition.org/cei/7253

DOI : $10.4000 /$ cei.7253

ISSN : 2260-779X

\section{Éditeur}

UGA Éditions/Université Grenoble Alpes

Édition imprimée

ISBN : 978-2-37747-225-3

ISSN : 1770-9571

Référence électronique

Jean Sénié, «Ippolito II d'Este, les artistes et le royaume de France (1536-1563) », Cahiers d'études italiennes [En ligne], 31 | 2020, mis en ligne le 06 octobre 2020, consulté le 26 mars 2021. URL : http:// journals.openedition.org/cei/7253; DOI : https://doi.org/10.4000/cei.7253

Ce document a été généré automatiquement le 26 mars 2021

(c) ELLUG 


\section{Ippolito II d'Este, les artistes et le royaume de France (1536-1563)}

Ippolito II d'Este, gli artisti e il regno di Francia (1536-1563)

Ippolito II d'Este, Artists and the Kingdom of France (1536-1563)

\section{Jean Sénié}

« Io essendo già expedito di qua, et attendesi a fare gran ciera a Fontanableo, prima di partirmi volsi vedere certe bellissime statue di bronzo che ivi in una camera S. $\mathrm{M}^{\text {tà }}$ Christianissima facea fare

et quali sono presso che finite et essendo io in detta camera mi sopragiunse il Re Christianissimo che a brazzo teniva Madama d'Etampes con solo il

$\mathrm{R}^{\mathrm{mo}} \mathrm{S}^{\mathrm{r}}$ Carle Nostro seco, Mons ${ }^{\mathrm{r}} \mathrm{d}^{\text {'Annibo, una }}$ sorella di detta Madama di Etampes et due damiselle, dove stettero buon pezzo a ragionare, et $\mathrm{S}$. $\mathrm{M}^{\text {tà }}$ mostrava alla predetta Madama d'Etampes una venere, come ella era di bel corpo perfettamente formata, la quale non disse altre, ma sorridendo intrò subito in una camera con le altre donne a scaldarsi et il Re Christianissimo restò col S $\mathrm{S}^{\mathrm{r}} \mathrm{Car}^{\mathrm{le}}$ al quanto a divisare di quelle

figure ${ }^{1}$."

\section{Introduction}

1 Une lettre de l'ambassadeur ferrarais, Alfonso Calcagnini, adressée au duc de Ferrare, Ercole II d'Este relatant une discussion entre François I $^{\text {er }}$ et d'Ippolito II d'Este au sujet d'une Vénus, permet de constater l'existence des relations entre la cour de France, le cardinal de Ferrare et les artistes italiens. En effet, les modèles de ces statues sont vraisemblablement des antiques dont le cardinal de Ferrare était un grand amateur ${ }^{2}$. Ce 
dernier peut d'ailleurs être considéré comme l'un des instigateurs des collections d'antiques en France dans la première moitié du XvI siècle. Plus précisément, Adolfo Venturi a proposé de voir dans la Vénus de Cnide, apportée d'Italie par Francesco Primaticcio, le modèle de cette Vénus si attrayante ${ }^{3}$. Il suffit de rappeler les liens de l'artiste avec les Guise, parents d'Ippolito par sa nièce Anne d'Este, épouse de François de Guise, pour voir qu'autour du prélat s'organise une intense circulation d'œuvres d'art et d'artistes venus d'Italie ${ }^{4}$. Or, la discussion entre François I ${ }^{\text {er }}$ et Ippolito II d'Este vient rappeler que le cardinal italien se situe à l'interface des cours française et italienne - alors en pleine rencontre - et qu'il est un des promoteurs de cette «présence italienne » mais aussi un de ses bénéficiaires ${ }^{5}$.

2 Depuis les travaux d'Anne Le Coq, les historiens connaissent le rôle joué par François I ${ }^{\text {er }}$ dans la construction d'un imaginaire politique moderne, fondé sur une nouvelle symbolique ${ }^{6}$. Or, Ippolito II d'Este contribue à l'élaboration de cette grammaire du pouvoir, notamment en faisant venir des artistes et théoriciens de l'art italien en France ${ }^{7}$. Au-delà d'artistes italiens renommés, les livres de comptes, conservés à l'Archivio di Stato de Modène, révèlent les noms d'artistes émiliens faisant partie de la suite du cardinal. Il est d'ailleurs notable que ce rôle de passeur du cardinal d'Este ne soit pas à sens unique puisqu'il ramène aussi avec lui, quand il rentre en Italie en 1549, des artistes français, tels Laurent Tibault ou Claude Marcel, orfèvres à Paris à l'enseigne des Trois Villages, Jacques Martin, orfèvre à l'enseigne du Cheval Cabré, Philippe Lascier, orfèvre à l'enseigne du Moulinet ou encore Lambert Hotman, orfèvre à l'enseigne du Panier vert ${ }^{8}$.

3 À un deuxième niveau, Ippolito II d'Este instaure avec les mécènes français en général, et les cardinaux français en particulier, "un dialogue, une émulation et une compétition " pour reprendre l'heureuse expression de Flaminia Bardati ${ }^{9}$. C'est dans cette interaction qu'Ippolito II d'Este contribue à la diffusion et à la réinterprétation de modèles artistiques italiens dans le royaume de France. À cet égard, il faut relever que les ambassadeurs de Ferrare présents à la cour de France, dans les rapports détaillés qu'ils envoient au duc de Ferrare sur son frère, insistent sur sa passion pour les statues antiques $^{10}$. Celle-ci constitue le régal de ses vieux jours dans sa villa de Tivoli ${ }^{11}$.

4 L'étalage des produits de son mécénat par le prélat s'inscrit dans le cadre d'un jugement esthétique qu'il partage avec les ambassadeurs ferrarais. Pour eux, le royaume de France est la terre du gothique, visible sous toutes ses formes, et il n'aurait pas profité des bienfaits de la redécouverte de l'Antiquité. Pour les Italiens réunis autour du cardinal de Ferrare, les traditions françaises ne sont belles que lorsqu'elles se font les épigones des accomplissements artistiques italiens. En ce sens, l'attitude de ce groupe italien devant la renovatio française qu'ils observent est contrastée. En dernière instance, c'est la question de la possibilité d'un retour aux sources à l'échelle européenne qui est passée au crible du scepticisme d'observateurs italiens. Si les Français ne sont plus les «Barbares » de Guicciardini, ils n'en manquent pas moins de finesse pour l'ambassadeur Giulio Alvarotti.

5 Pour autant, Ippolito a besoin de l'appui de la couronne de France pour s'assurer une place de premier plan à la Curie et dans le jeu diplomatique des familles princières italiennes. Il voit aussi dans le royaume de France la possibilité d'obtenir une indépendance économique et une surface politique, indispensables à l'acquisition de son autonomie par rapport au duc de Ferrare. L'enjeu est de dépasser sa condition initiale de cardinal «de famille » pour obtenir un rôle politique à l'interface des trois 
puissances que sont le royaume de France, les États pontificaux et le duché de Ferrare ${ }^{12}$. Parmi les atouts qui s'offrent à lui, l'attrait de la cour de France pour les objets d'art et les compétences artistiques des hommes de la Cour, s'avère primordial. Mais ce mécénat s'étend en réalité à tous les domaines artistiques qui fleurissent dans la Ferrare de la Renaissance ${ }^{13}$. En ce sens, Ippolito II d'Este apparaît comme le parangon du prélat italien issu d'une dynastie familiale. Cependant, l'image de lui que ce déploiement de fastes contribue à forger comporte également un versant négatif : de splendides, le mécénat du cardinal et les artistes qui l'entourent en viennent à être assimilés aux stéréotypes anti-italiens alors en voie d'élaboration. À tel point que, lors de son dernier séjour en France en tant que légat a latere, entre 1561 et 1563, Ippolito II d'Este devient la cible de libelles protestants mais aussi catholiques. Cette tradition est d'ailleurs appelée à une durable prospérité, puisque ces stéréotypes se retrouveront chez Lucien Romier dans son portrait à charge du cardinal de Ferrare ${ }^{14}$.

6 Ainsi, le cardinal de Ferrare se fonde sur l'image qu'il a des Français pour adapter son comportement à la cour et se distinguer auprès du roi de France, il contribue en retour à l'élaboration d'une image des Italiens en général, et plus particulièrement, du prélat italien de la Renaissance, qui fonctionne selon un principe symétrique. En somme, la présence à la cour du cardinal d'Este rejoint le thème plus global d'un particularisme italien en train de se figer dans les discours ${ }^{15}$. Or, cette nouvelle sémantique connaît une diffusion européenne, tout au moins dans le monde protestant, relayant les vitupérations de Poissy. La présence des artistes accompagnant Ippolito II d'Este pose ainsi la question du rapport entre un groupe italien circonscrit et le royaume de France, tant sur le plan de la diffusion artistique de modèles culturels que sur celui de l'émergence d'un discours stéréotypé sur des catégories ciblées d'hommes issus de la péninsule italienne.

7 Après avoir présenté les enjeux politiques du mécénat d'Ippolito II d'Este, des exemples d'artistes provenant des différents champs sont proposés pour illustrer à la fois la tentative du cardinal de se placer à l'interface du royaume de France et de la péninsule italienne et les différences entre les parcours d'artistes. Les pamphlets qui l'accueillent à son retour en France font état du retournement qui s'est opéré à l'aube des guerres de Religion au niveau de l'image du prélat italien, renversement dont d'autres Italiens présents à la cour firent également les frais.

\section{Ippolito II d'Este, un mécénat au service d'un parcours curial}

8 Le 17 mars 1541, Ippolito II d'Este offre à François I ${ }^{\text {er }}$ une aiguière et un bassin réalisés par l'orfèvre Benvenuto Cellini ${ }^{16}$. Le 4 septembre 1541, il juge bon d'offrir au roi une épée ramenée de Ferrare par Ludovico da Thiene ${ }^{17}$. C'est encore lui qui fait envoyer, depuis la péninsule italienne, des chevaux à Henri II pour lui en faire don, en décembre $1552^{18}$. La société $\mathrm{du} \mathrm{XVI}^{\mathrm{e}}$ siècle fonctionne selon des pratiques de don et, a fortiori, la société de cour ${ }^{19}$. Il n'est pas incongru de voir le cardinal de Ferrare multiplier les dons aux rois de France et à leur entourage ${ }^{20}$. Néanmoins, ceux-ci ne peuvent manquer de frapper tant par leur ampleur que par leur caractère résolument artistique $^{21}$. 
9 Le cardinal a, en effet, senti qu'il pouvait bénéficier, à son arrivée dans le royaume des lys, de la différence entre les modèles artistiques italien et français. Il l'expose d'ailleurs à son frère dans une lettre du 16 octobre 1546, soit dix ans après sa venue en France :

Quanto al designo poi che la mi domanda de la mia casa di Fontanableo V.Ex ${ }^{\text {tia }}$ sappi che ella è assai manco in fatti di quel ch'ella ha per avventura il nome, et quel che la dee far forse, nominar per bella, credo che sia più tosto per esser fatta nel luogo dove è et dove par che sia più di quel che vi convegneria. Et per esservisi osservato anco un poco più le misure et ordini dell'architettura così nel Francese come in quel che ci è dell'Italiano, che non si sogliano così avvertire et osservare in quelle di questi paesi, che perché in effetto sia cosa segnalata né notabile. Et Ms. Bastian Serlio che ne è stato l'architetto, l'havea messa in un suo libro d'architettura, che ha ultimamente fatto et vuole far stampare et io ne l'ho fatta levare, parendomi che'l vederla in disegno fusse per levarle più tosto che per darle punto di riputatione. Ma se V.Ex. la vorrà pur qual ella è, non mancherò d'ubidirla così in questo come in quel tutto che la mi commanderà sempre et gliela manderò, il che anco non saprei far per adesso per non esser in luogo commodo ${ }^{22}$.

La demeure construite par l'architecte Sebastiano Serlio démontre combien il est possible d'acquérir de renommée à la cour de France, en mettant en avant les réalisations d'artistes italiens dont cette cour est alors avide ${ }^{23}$. Ippolito II d'Este entend bénéficier de son positionnement dans le champ artistique, notamment du décalage entre les canons français et les canons d'Italie, pour en récolter les fruits dans le champ politique et religieux, avec l'obtention de bénéfices ecclésiastiques ${ }^{24}$. Après le chapeau de cardinal obtenu en 1539, il reçoit, outre l'archevêché de Lyon et l'abbaye SaintMédard de Soissons, dont il est déjà le titulaire, l'abbaye de Jumièges en septembre 1539, celle de Chaalis en février 1541, pour ne citer que les premiers bénéfices obtenus par la faveur royale ${ }^{25}$.

11 Pour accomplir son objectif, Ippolito II d'Este dispose de plusieurs moyens. Le plus simple, et le plus direct, consiste à faire des dons d'œuvres provenant d'Italie, comme c'est le cas avec les marbres et les statues antiques. Il cherche aussi à valoriser les artistes venus d'Italie, dont il a parfois facilité le voyage depuis la péninsule, et qui se retrouvent à son service. Il suffit de penser à des poètes comme Luigi Alamanni ou Gabriele Cesano qui «chaque jour, après le dîner, lit Dante à madame la Dauphine, à madame Margherita et à Monseigneur d'Orléans » et que l'ambassadeur ferrarais décrit discutant avec Sebastiano Serlio, en mai 1546, des principes de la castramétation tels qu'exposés par Polybe dans ses Histoires ${ }^{26}$. On retrouve aussi des peintres comme le Primaticcio, des mosaïstes comme Giovanni Magnanini et des orfèvres et sculpteurs comme Cellini. C'est encore le cas du musicien Francesco Della Viola, au service du cardinal de Ferrare dans les années 1540 et $1550^{27}$. Enfin, le prélat contribue à la diffusion de modèles artistiques. Cela se fait de manière naturelle, comme dans le cas du Primaticcio passant de sa clientèle à celle de ses parents, les Guise. Le cardinal de Ferrare marque aussi l'abbaye de Chaalis de son empreinte, en procédant à un réaménagement ambitieux, notamment dans les jardins ${ }^{28}$. En ce lieu, l'architecte Sebastiano Serlio donne toute la mesure de son talent. Il reste aujourd'hui un portail ouvrant un mur crénelé abritant le jardin, reprenant le dessin des bossages rustiques que l'on retrouve dans les portails romains de Bramante ${ }^{29}$. Il connait immédiatement un grand succès comme l'atteste le rez-de-chaussée de la façade de l'hôtel Carnavalet ${ }^{30}$.

12 Le cardinal acquiert ainsi un rôle de médiateur entre l'Italie et le royaume de France. Cette place de médiateur lui permet rapidement de jouer aussi un rôle sur le plan politique $^{31}$. Il devient une personne de référence dans le champ de la diplomatie 
italienne, où il agit au service du roi de France, sans pour autant oublier ses propres intérêts. Ippolito II d'Este tient ainsi table ouverte pour les prélats italiens présents à la cour de France ${ }^{32}$. Il parvient de cette façon à transformer la faveur royale en capital politique. Ayant constaté l'appétence royale pour l'art et les artistes italiens, il investit ce créneau pour consolider sa position politique ${ }^{33}$. Le prélat est un des organisateurs, avec Catherine de Médicis, du groupe d'artistes italiens bellifontains ${ }^{34}$. C'est vers eux qu'il faut désormais porter le regard, auteurs d'une synthèse inédite des traditions artistiques des deux côtés des Alpes, formant un spectre qui va de l'hybridation culturelle à la réaffirmation d'une séparation artistique nette entre les deux entités géographiques.

\section{Parcours d'artistes de la suite du cardinal de Ferrare}

13 Ippolito II d'Este emploie des artistes italiens dans le royaume de France. Il peut aussi bien s'être lié avec eux sur place que les avoir fait venir d'Italie. Afin d'éclairer ce propos, le mieux est encore de prendre des exemples présentant des artistes de la suite du cardinal et d'étudier la conception qu'ils se sont faite de la différence entre Italiens et Français.

Les Italiens qui se trouvent à Fontainebleau, formant une communauté soudée et fermée, sont d'abord soumis au problème de la langue, "en ceste solitude de Fontainebleau » pour reprendre les mots de Sebastiano Serlio ${ }^{35}$. Benvenuto Cellini, qui reste à Paris et en marge des Italiens bellifontains, rappelle dans son autobiographie que François I ${ }^{\text {er }}$ parlait avec Ippolito II d'Este en français puis ajoute «voltosi a me mi parlò in italiano $0^{36} \%$.

15 L'artiste Francesco Primaticcio, entre autres, se retrouve dans cette configuration. Il est aussi bien au service de François $I^{\text {er }}$ que d'Ippolito II d'Este, pour lequel il embellit de fresques sa chapelle abbatiale de Chaalis ou décore les bains du Grand Ferrare ${ }^{37}$. Même si l'artiste n'a pas la même proximité avec le prélat que Benvenuto Cellini ou Sebastiano Serlio, il n'en est pas moins entré en relation le cardinal de Ferrare. C'est d'ailleurs par l'entremise de la famille d'Este que le peintre noue d'étroites relations avec le cardinal Charles de Lorraine, beau-frère d'Anne d'Este, la nièce d'Ippolito II d'Este. Le Primaticcio participe d'une période de bouillonnement où les modèles artistiques importés de la péninsule italienne se diffusent et sont réinterprétés dans des synthèses originales.

Le cas de Sebastiano Serlio montre les difficultés des artistes italiens à trouver leur public en France. Le principal appui de Serlio à la cour reste Ippolito II d'Este. Les liens de Serlio avec la maison d'Este sont anciens. Toutefois, c'est le cardinal qui est son principal commanditaire et, à ce titre, son principal soutien. L'architecte semble dessiner pour lui son premier édifice en France et il n'est pas à exclure que ce soit le prélat qui lui ait procuré sa situation en France. Le prélat lui offre un logement au Grand Ferrare ${ }^{38}$. Les années 1542-1545 sont parmi les plus fécondes de son activité française. L'année 1545, en particulier, est celle qui voit l'édition franco-italienne du Primo et du Secondo Libro, où les textes des deux langues sont disposés en vis-à-vis. En ce sens, il est possible de parler d'une entreprise de diffusion d'un savoir, en l'occurrence celui de son maître Baldassare Peruzzi, dans le royaume de France. De même, ce sont les années où il dirige les travaux du «Grand Ferrare » et de l'abbaye de Chaalis ${ }^{39}$. 

l'arrivée sur le trône d'Henri II. Le nouveau roi lui préfère un architecte français, Philibert De L'Orme. Il est toutefois intéressant de noter que celui-ci est un familier de l'architecture italienne qu'il a étudiée à Rome. Le départ du cardinal le prive d'un soutien dans sa quête de reconnaissance artistique et le laisse amer. Pour Sabine Frommel, l'échec relatif de Sebastiano Serlio traduit l'inadéquation entre le projet artistique d'un Italien et les aspirations esthétiques françaises, les deux ne parvenant qu'occasionnellement à se rencontrer ${ }^{40}$. Néanmoins le «Grand Ferrare ", de même que le château d'Ancy-le-Franc, forment un exemple réussi «d'imbrications typologiques ${ }^{41} »$. tout comme avec l'ensemble des personnes qui croisent sa route, celle du sculpteur Benvenuto Cellini. Dans le cas de l'irascible orfèvre, il est intéressant de voir qu'Ippolito II d'Este, agissant sur commande royale, facilite sa sortie des prisons romaines. Il se sert ensuite des dons de l'orfèvre pour arriver les bras chargés de présents pour François $\mathrm{I}^{\mathrm{er}}$, à commencer par un pot et un bassin ${ }^{42}$. C'est aussi le cardinal qui oriente l'orfèvre vers le roi pour son projet de salière, si grandiose selon Ippolito II d'Este qu'il ne pourrait convenir qu'à un roi. Luigi Alamanni et Gabriele Cesano lui ont d'ailleurs proposé chacun un programme iconographique avant de se voir congédier par Benvenuto Cellini qui leur répond, devant le prélat de la maison d'Este, que «sarà mia opera e mia invenzione ${ }^{43}$ ». Ippolito est conscient du caractère éruptif de Cellini, mais il entend aussi profiter de son talent, tout au moins tant que François $\mathrm{I}^{\text {er }}$ tolère le comportement du Florentin ${ }^{44}$.

Ces exemples montrent comment le mécénat artistique d'Ippolito II d'Este, selon une stratégie de la magnificence, entraîne des artistes italiens à participer aux redéfinitions des canons artistiques français. Pour autant, cette image va être entièrement inversée lors de son dernier séjour en France.

\section{Le retournement du discours (1561-1572)}

La venue du légat a latere fait l'objet de vives contestations dans le royaume de France, en particulier chez les protestants. Au moment du colloque de Poissy, Théodore de Bèze porte des jugements méprisants sur le cardinal, notamment en raison de sa frivolité ${ }^{45}$. Ces arguments se retrouvent dans la première histoire des affrontements religieux, les Commentaires de l'État de la religion de Pierre de La Place, parue en 1565. La richesse du cardinal, ses nombreux bénéfices sont autant de défauts jouant en sa défaveur ${ }^{46}$. Toutefois, ils sont accrus et cristallisés par son appartenance à une famille princière italienne. Il revêt désormais l'habit de l'étranger, source de divisions. L'ensemble des traits de la polémique protestante sont repris dans les Mémoires de Condé avec un texte, vraisemblablement rédigé per le protestant Jacques Spifame de Brou, où le cardinal de Ferrare est traité avec Louis de Guise de «belle[s] statue[s] muette[s] $]^{47} »$. Le renversement est sanctionné par l'inversion des valeurs se rapportant à son mécénat, originellement source de renommée et désormais reflet de son incompétence dans le texte de Spifame de Brou.

21 Or ce discours protestant sur le cardinal de Ferrare se répand en Europe dans les régions de confessions calvinistes. Il contribue à forger l'image du prélat italien, corrompu et jouisseur. L'idée d'une légation ratée car attribuée à un homme 
incompétent se retrouve également chez des catholiques, en particulier chez les "politiques ». Jacques-Auguste du Thou décrit dans son Histoire universelle une légation inefficace et incapable de servir de médiation, sous-entendant que la personnalité du cardinal a probablement été un facteur aggravant. Là encore, le cardinal se voit brossé sous les traits de l'ultramontain incapable de saisir les besoins d'un royaume caractérisé par sa foi gallicane ${ }^{48}$.

Le retournement du discours sur les Italiens a été étudié plus amplement par Henry Heller dans son ouvrage Anti-Italianism in Sixteenth century France ${ }^{49}$. L'éclatement des guerres de Religion apparait ainsi comme un moment de cristallisation du sentiment xénophobe à l'encontre des Italiens, qui culmine avec la Saint-Barthélemy ${ }^{50}$. Le fonctionnement même du système de la faveur royale, qui nécessitait la mise en avant de leur munificence par les Italiens, finit par se retourner contre eux quand cette munificence est vue, à l'aune des discours polémiques, comme un étalage frivole de richesse justifiant la violence punitive dont ils font l'objet. Or les propagateurs de l'image négative du cardinal d'Este sont aussi ceux qui, plus généralement, contribuent à figer les lieux communs des discours anti-italiens.

Comme d'autres Italiens présents à la cour, le cardinal apparaît ainsi « subtil et fin ", expert en «l'art de la tromperie ». De même, tout comme d'autres prélats italiens, il accaparerait les bénéfices ecclésiastiques français, affamant le clergé français pour vivre dans le luxe ${ }^{51}$. Les actions d'Ippolito II sont alors interprétées au prisme de d'une nouvelle rhétorique anti-italienne. Les tensions entre italianisme et anti-italianisme augmentent, stigmatisant les vertus d'hier, alors que "résistance culturelle et résistance politique se confond[ent $]^{52} »$. Les Italiens devenant alors, tout du moins pour partie, une structure rhétorique dans les discours entendant définir l'identité des Français.

\section{Conclusion}

Étudier les stratégies curiales d'Ippolito II d'Este permet de voir en quoi la culture des membres de l'élite italienne constitue une ressource dans l'optique d'une insertion dans le royaume de France. La trajectoire biographique du cardinal de Ferrare montre, en outre, que la réciproque peut constituer un atout dans son affirmation politique à la Curie. Toutefois, à l'aube des guerres de Religion, à un moment où se recomposent les clivages confessionnels et politiques, le marqueur distinctif qu'est cette culture italienne peut devenir un stigmate, utilisé à des fins de propagande.

À un premier niveau, l'évolution des représentations du prélat et de sa suite doit être comprise comme une manifestation de la tension entre italianisme et anti-italianisme. Toutefois, en déplaçant le problème, outre son italianité, il est aussi fait le reproche au fils de Lucrèce Borgia d'être un homme de la Renaissance. En ce sens, il appartient à une Europe en train de disparaître devant l'émergence des tensions confessionnelles et politiques, caractéristiques d'une nouvelle configuration européenne, celle des États modernes ${ }^{53}$.

La comparaison avec son neveu, Luigi d'Este, à son tour cardinal-protecteur de la France, éclaire cette prise de conscience du caractère plus difficilement franchissable des démarcations culturelles. Luigi d'Este ne cherche pas à promouvoir en France un art italien ou des artistes italiens. Il joue au contraire sur l'avantage que peut tirer le roi de France d'un Italien fin connaisseur des arcanes du Saint-Siège. Pour autant, il 
continue à accueillir dans la villa Tivoli des Français de passage, parmi lesquels Montaigne. Il poursuit ainsi le rôle d'intermédiaires culturels des cardinaux d'Este entre la France et l'Italie.

\section{BIBLIOGRAPHIE}

AsHBY Thomas, « The Ville d'Este at Tivoli and the Collection of Classical Sculptures Which It Contained ", Archaeologia or Miscellaneous Tracts Relating to Antiquity, vol. 61, n 1, 1908, p. 219-256. BABELON Jean-Pierre, « Du grand Ferrare à Carnavalet, naissance de l'hôtel classique », Revue de l'art, vol. 41, 1978, p. 83-108.

BALSAMO Jean, Les Rencontres des Muses. Italianisme et anti-italianisme dans les Lettres françaises de la fin $d u X^{X} I^{e}$ siècle, Genève, Slatkine, 1992.

BARDATI Flaminia, « Ippolito II d'Este e i cardinali francesi: dialogo, emulazione, competizione », dans M. Cogotti et F. P. Fiore (éds), Ippolito II d'Este, cardinale, principe, mecenate, Rome, De Luca Editori d'Arte, 2013, p. 73-89.

BARDATI Flaminia, Hommes du roi et princes de l'Église romaine. Les cardinaux français et l'art italien (1495-1560), Rome, École française de Rome, 2015.

BÈzE Théodore de, Histoire ecclésiastique des Églises réformées au Royaume de France, t. I, G. Baum, É. Cunitz et R. Reuss (éds), Nieuwkoop, B. de Graaf, 1974.

BOUCHER Jacqueline, Présence italienne à Lyon à la Renaissance. Du milieu du XVe à la fin du XVI siècle, Lyon, Éditions LUGD, 1994.

BOUCHER Jacqueline, Société et mentalités autour de Henri III, Paris, Honoré Champion, 2007.

COESTER Christiane, Schön wie Venus, mutig wie Mars. Anna d'Este, Herzogin von Guise und von Nemours (1531-1607), Munich, Oldenbourg, 2007.

COOPER Richard, Roman Antiquities in Renaissance France (1515-65), Farnham, Ashgate, 2013.

Dubost Jean-François, La France italienne, XVI ${ }^{e}$-XVII ${ }^{e}$ siècle, Paris, Aubier, 1997.

Dufour Alain et MeYlan Henri (éds), Correspondance Théodore de Bèze, t. III, Genève, Librairie Droz, 1963.

FERRUTI Francesco, « La villa d'Este a Tivoli e la collezione di sculture classiche che conteneva (di Thomas Ashby) ", Atti e Memorie della Società Tiburtina di Storia e d'Arte, vol. LXXXII, 2009, p. 169-278.

FERRUTI Francesco, « La collezione di sculture antiche di Ippolito II d'Este: su alcuni esemplari », dans M. Cogotti et F. P. Fiore (éds), Ippolito II d'Este, cardinale, principe, mecenate, Rome, De Luca Editori d'Arte, 2013, p. 367-390.

FIRPO Massimo, « Le Cardinal », dans E. Garin (dir.), L'homme de la Renaissance, Paris, Seuil, 2002 (1990), p. 97-107. 
Frommel Sabine, Sebastiano Serlio. Architecte de la Renaissance, trad. Yves Pauwels, Paris, Gallimard, 2002.

Frommel Sabine (dir.), avec la collab. de Flaminia BARDATI, Primatice architecte, Paris, Picard, 2005.

FROMMEL Sabine, « Hippolyte d'Este à Chaalis : architecture projetée, architecture illusionniste », Les Monuments Piot, vol. 87, 2008, p. 143-172.

GRODECKI Catherine, Documents du Minutier central des notaires de Paris. Histoire de l'art au XVIe siècle (1540-1600). I : Architecture, vitrerie, menuiserie, tapisserie, jardins, Paris, Archives nationales, 1985.

GuERZONI Guido, «"Familia”, "corte”, “casa”. The Este Casa in Fifteenth-Sixteenth Century ", dans W. Paravicini (éd.), La cour de Bourgogne et l'Europe. Le rayonnement et les limites d'un modèle culturel, Actes du colloque international (Paris, 9-11 octobre 2007), Ostfilden, Jan Thorbecke, 2013, p. 515-541.

HELLER Henry, Anti-Italianism in Sixteenth-Century France, Toronto, University of Toronto Press, 2003.

HOLLINGSWORTH Mary, " Coins, Cloaks and Candlesticks: The Economics of Extravagance », dans M. O'Malley et E. Welch (éds), The Material Renaissance, Manchester, Manchester University Press, 2007, p. 260-287.

JAMES François-Charles, «L'Hôtel du cardinal de Ferrare d'après un document inédit », dans A. Chastel (éd.), Actes du colloque international sur l'art de Fontainebleau (18-20 octobre 1972), Paris, Éditions du CNRS, 1975, p. 35-37.

JESTAZ Bertrand, «L'exportation des marbres de Rome de 1535 à 1571 », Mélanges d'archéologie et d'histoire, vol. 75, $n^{\circ} 2,1963$, p. 415-466.

JESTAZ Bertrand, « Les Italiens à Fontainebleau », Künstlerischer Austauch, vol. 28, 1992, p. 93-104. JESTAZ Bertrand, «Benvenuto Cellini et la cour de France (1540-1545) ", Bibliothèque de l'École des Chartes, vol. 161, 2003, p. 71-132.

LA Place Pierre de, Commentaires de l'estat de la religion et république soubs les rois Henry et François seconds et Charles neufiesme, s. 1., 1565.

LECOQ Anne-Marie, François I Ir. Imaginaire symbolique et politique à l'aube de la Renaissance française, Paris, Macula, 1987.

LE RouX Nicolas, « La Saint-Barthélemy des Italiens n'aura pas lieu : un discours envoyé à Catherine de Médicis en 1573 », dans B. Barbiche, J.-P. Poussou et A. Tallon (éds), Pouvoirs, contestations et comportements dans l'Europe moderne : mélanges en l'honneur du professeur Yves-Marie Bercé, Paris, PUPS, 2005, p. 165-183.

LESTOCQUOY Jean (éd.), Correspondance des nonces en France : Carpi et Ferrerio. 1535-1540, Rome / Paris, Presses de l'Université grégorienne / De Boccard, 1961.

LESTOCQuoy Jean (éd.), Correspondances des nonces en France : Dandino, Della Torre et Trivultio (1546-1551), Paris / Rome, De Boccard / Presses de l'Université grégorienne, 1966.

MEISS-EVEn Marjorie, « “The Guise Italianized?” The Role of Italian Merchants, Intermediaries and Experts in Ducal Consumption in the Sixteenth Century ", dans J. Munns, P. Richards et J. Spangler (éds), Aspiration, Representation and Memory: The Guise in Europe 1506-1688, Farnham, Ashgate, 2015, p. 47-60. 
Michon Cédric, " Mécénat et politique : le cas des prélats français et anglais à la Renaissance ", dans F. Lemerle, Y. Pauwels et G. Toscano (éds), Les Cardinaux de la Renaissance et la modernité artistique, Villeneuve-d'Ascq, Septentrion, 2009, p. 23-37.

Michon Cédric, « Hippolyte d'Este (1509-1572) », dans Id. (dir.), Les Conseillers de François I ${ }^{\text {er }}$, Rennes, PUR, 2011, p. 527-532.

OcchiPINTI Carmelo, « Il "camerino" e la "galleria" nella Villa d'Este a Fontainebleau ("Hôtel de Ferrare") », Annali della Scuola Normale Superiore di Pisa, s. IV, vol. II, 1997, p. 601-635.

OcchiPINTI Carmelo, « La Villa d'Este a Fontainebleau e le sue "stufette”. Documenti su Serlio e il Cardinale di Ferrara », Prospettiva, vol. 89-90, 1998, p. 169-183.

OcchIPINTI Carmelo, Carteggio d'arte degli ambasciatori estensi in Francia (1535-1553), Pise, Scuola Normale Superiore, 2001.

PelLegrini Marco, « Naissance d'une typologie : le cardinal italien de famille princière à l'époque de la première Renaissance ", dans M. Maillard-Luypaert, A. Marchandisse et B. Schnerb (éds), Évêques et cardinaux princiers et curiaux (XIVe-début XVI siècle) : des acteurs du pouvoir, Turnhout, Brepols, 2017, p. 217-226.

PENZI Marco, «L'Italia e gli italiani visti dai Francesi alla fine del XVI secolo », dans R. Russi (éd.), L'Italia vista dagli altri, Atti del I Convegno internazionale (Banka Luka, 12-13 giugno 2009), Florence, Franco Cesati editore, 2010, p. 81-100.

Petey-Girard Bruno, Polizzi Gilles et Tran Trung (éds), François I ${ }^{e r}$ imaginé, Actes du colloque de Paris organisé par l'association Renaissance-Humanisme-Réforme et par la Société française d'étude du seizième siècle (Paris, 9-11 avril 2015), Genève, Librairie Droz, 2017.

ROMIER Lucien, Les origines politiques des guerres de Religion, t. I, Paris, Perrin, 1913.

ROMIER Lucien, Catholiques et huguenots à la cour de Charles IX : les états généraux d'Orléans, le colloque de Poissy, le « concordat » avec les protestants, le massacre de Vassy (1560-1562), Paris, Perrin, 1924 ( $2^{\mathrm{e}}$ éd.).

SERLIO Sebastiano, Il primo libro d'Architettura, Paris, De l'imprimerie de Jehan Barbé, 1545.

SMITH Marc H., « Complots, révoltes et tempéraments nationaux : Français et Italiens au XVI ${ }^{\mathrm{e}}$ siècle », dans Y.-M. Bercé et E. Fasano Guarini (éds), Complots et conjurations dans l'Europe moderne, Actes du colloque international organisé à Rome (30 septembre-2 octobre 1993), Rome, École française de Rome, 1996, p. 93-115.

TALLON Alain, L'Europe au XVI siècle. États et relations internationales, Paris, PUF, 2010.

Tosini Patrizia, « Per una geografia dei pittori francesi a Roma, alla mèta del Cinquecento: committenti, opere, contesti », à paraître.

VENTURI Adolfo, «L'arte e gli Estensi: Ippolito II d'Este cardinale di Ferrara in Francia », Rivista europea. Rivista internazionale, vol. XXIV, 1881, p. 27-37.

VENTURI Adolfo, « Una visita artistica di Francesco I re di Francia », Archivio storico dell'arte, fasc. VIII-IX, 1889, p. 377-378.

Zemon-Davies Natalie, The Gift in Sixteenth-Century France, Oxford, Oxford University Press, 2000. 


\section{NOTES}

1. Archivio di Stato di Modena, Archivio segreto estense, Cancelleria, Sezione Estero, Carteggio ambasciatori, Francia, b. 19 [désormais ASMo, Carteggio ambasciatori, Francia], Alfonso Calcagnini à Ercole II d'Este, 23 décembre. Pour resituer cette visite dans le cadre du collectionnisme d'antiques à la cour de France, voir R. Cooper, Roman Antiquities in Renaissance France (1515-65), Farnham, Ashgate, 2013, p. 141-142 et 145.

2. Ibid., p. 134.

3. A. Venturi, "Una visita artistica di Francesco I re di Francia ", Archivio storico dell'arte, fasc. VIII-IX, 1889, p. 377.

4. Sur le rôle du Primaticcio comme agent artistique d'Ercole II d'Este, voir ASMo, Carteggio ambasciatori, Francia, b. 25, Giulio Alvarotti à Ercole II d'Este, Melun, 3 janvier 1548, reproduite dans C. Occhipinti, Carteggio d'arte degli ambasciatori estensi in Francia (1535-1553), Pise, Scuola Normale Superiore, 2001, p.191. Voir aussi S. Frommel (dir.), avec la collab. de F. Bardati, Primatice architecte, Paris, Picard, 2005, p. 323, Francesco Primaticcio à Simon Renard, Fontainebleau, 28 octobre 1549. Sur les liens de Francesco Primaticcio avec les Guise, voir en dernier lieu M. Meiss-Even, "“The Guise Italianized?" The Role of Italian Merchants, Intermediaries and Experts in Ducal Consumption in the Sixteenth Century ", dans J. Munns, P. Richards et J. Spangler (éds), Aspiration, Representation and Memory: The Guise in Europe 1506-1688, Farnham, Ashgate, 2015, p. 50-53. Pour Christiane Coester, le rôle d'Anne d'Este dans l'entrée du Primaticcio au service des Guise ne doit pas être surévalué. C. Coester, Schön wie Venus, mutig wie Mars. Anna d'Este, Herzogin von Guise und von Nemours (1531-1607), Munich, Oldenbourg, 2007, p. 126.

5. J. Boucher, Présence italienne à Lyon à la Renaissance. Du milieu du XV à la fin du XVI ${ }^{e}$ siècle, Lyon, Éditions LUGD, 1994.

6. A.-M. Lecoq, François I ${ }^{e r}$. Imaginaire symbolique et politique à l'aube de la Renaissance française, Paris, Macula, 1987. Voir aussi B. Petey-Girard, G. Polizzi et T. Tran (éds), François I Ir imaginé, Actes du colloque de Paris organisé par l'association Renaissance-Humanisme-Réforme et par la Société française d'étude du seizième siècle (Paris, 9-11 avril 2015), Genève, Librairie Droz, 2017.

7. P. Tosini, «Per una geografia dei pittori francesi a Roma, alla mèta del Cinquecento: committenti, opere, contesti », à paraître.

8. A. Venturi, «L'arte e gli Estensi: Ippolito II d'Este cardinale di Ferrara in Francia », Rivista europea. Rivista internazionale, vol. XXIV, 1881, p. 35.

9. F. Bardati, «Ippolito II d'Este e i cardinali francesi: dialogo, emulazione, competizione », dans M. Cogotti et F.P. Fiore (éds), Ippolito II d'Este, cardinale, principe, mecenate, Rome, De Luca Editori d'Arte, 2013, p. 73-89.

10. Voir, par exemple, ASMo, Carteggio ambasciatori, Francia, b.29, Giulio Alvarotti à Ercole II d'Este, Reims, 22 novembre 1552.

11. T. Ashby, "The Ville d'Este at Tivoli and the Collection of Classical Sculptures Which It Contained ", Archaeologia or Miscellaneous Tracts Relating to Antiquity, vol. 61, n 1, 1908, p. 219-256 ; F. Ferruti, «La villa d'Este a Tivoli e la collezione di sculture classiche che conteneva (di Thomas Ashby) ", Atti e Memorie della Società Tirburtina di Storia e d'Arte, vol. LXXXII, 2009, p. 169-278 ; Id., «La collezione di sculture antiche di Ippolito II d'Este: su alcuni esemplari », dans M. Cogotti et F. P. Fiore (éds), Ippolito II d'Este, cardinale, principe, mecenate, ouvr. cité, p. 367-390. Sur les entreprises d'excavations d'Ippolito II d'Este, voir B. Jestaz, « L'exportation des marbres de Rome de 1535 à 1571 ", Mélanges d'archéologie et d'histoire, vol. 75, nº 2, Paris, De Boccard, 1963, p. $461-462, \mathrm{n}^{\text {os }} 72$ et 73 .

12. Sur la notion de cardinal «de famille», voir M. Pellegrini, « Naissance d'une typologie : le cardinal italien de famille princière à l'époque de la première Renaissance », dans M. MaillardLuypaert, A. Marchandisse et B. Schnerb (éds.), Évêques et cardinaux princiers et curiaux (XIVe-début XVI ${ }^{e}$ siècle) : des acteurs du pouvoir, Turnhout, Brepols, 2017, p. 217-226. 
13. Massimo Firpo rappelle l'importance de l'ostentation dans la suite du cardinal ainsi que la nécessité d'afficher sa prodigalité. M. Firpo, «Le Cardinal », dans E. Garin (dir.), L'homme de la Renaissance, Paris, Seuil, 2002 (1990), p. 97-107, ici p. 101-103.

14. L. Romier, Catholiques et huguenots à la cour de Charles IX: les états généraux d'Orléans, le colloque de Poissy, le "concordat» avec les protestants, le massacre de Vassy (1560-1562), Paris, Perrin, 1924, p. 222-224. Voir aussi Id., Les origines politiques des guerres de Religion, t. I, Paris, Perrin, 1913, p. 97 et 104 .

15. Pour la distinction entre "familia», «corte ", "casa» appliquée à la maison d'Este, voir G. Guerzoni, " "Familia", "corte”, "casa". The Este Casa in Fifteenth-Sixteenth Century », dans W. Paravicini (éd.), La cour de Bourgogne et l'Europe. Le rayonnement et les limites d'un modèle culturel, Actes du colloque international (Paris, 9-11 octobre 2007), Ostfilden, Jan Thorbecke, 2013, p. 515-541.

16. ASMo, Carteggio ambasciatori, Francia, b. 16, Carlo Sacrati à Ercole II d'Este, Blois, 17 mars 1541. 17. ASMo, Carteggio ambasciatori, Francia, b. 17, Lodovico da Thiene à Ercole II d'Este, Charlieu, 4 septembre 1541.

18. ASMo, Carteggio ambasciatori, Francia, b. 29, Giulio Alvarotti à Ercole II d'Este, Compiègne, 28 décembre 1552. Il s'agit d'une pratique courante puisqu'Ippolito informe son frère en 1547 que le roi a bien reçu et apprécié les chevaux que celui-ci lui a donnés. ASMo, Archivio segreto estense, Cancelleria, Sezione Estero, Casa e Stato, b. 148, Ippolito II d'Este à Ercole II d'Este, SaintGermain-en-Laye, 11 juillet 1547.

19. N. Zemon-Davies, The Gift in Sixteenth-Century France, Oxford, Oxford University Press, 2000.

20. C. Michon, "Mécénat et politique : le cas des prélats français et anglais à la Renaissance ", dans F. Lemerle, Y. Pauwels et G. Toscano (éds), Les Cardinaux de la Renaissance et la modernité artistique, Villeneuve-d'Ascq, Septentrion, 2009, p. 23-37.

21. Sur la variété des dons d'Ippolito II d'Este et leur fonctionnement en système, voir M. Hollingsworth, "Coins, Cloaks and Candlesticks: The Economics of Extravagance », dans M. O'Malley et E. Welch (éds), The Material Renaissance, Manchester, Manchester University Press, 2007, p. 260-287.

22. ASMo, Archivio segreto estense, Cancelleria, Sezione Estero, Casa e Stato, b. 147, Ippolito II d'Este à Ercole II d'Este, Mareilles, 16 octobre 1546.

23. Sur les débuts des travaux, voir Archives nationales, MC/ET/CXXII/1064, 3 mars 1542. Ce document est connu depuis F.-C. James, «L'Hôtel du cardinal de Ferrare d'après un document inédit », dans A. Chastel (éd.), Actes du colloque international sur l'art de Fontainebleau (18-20 octobre 1972), Paris, Éditions du CNRS, 1975, p. 35-37. Sur les premiers projets datant de 1542, voir aussi MC/ET/XIX/162, 23 décembre 1542, « devis de serrurerie ». C. Grodecki, Documents du Minutier central des notaires de Paris. Histoire de l'art au XVI siècle (1540- 1600). I: Architecture, vitrerie, menuiserie, tapisserie, jardins, Paris, Archives nationales, 1985, p. 247-248, «vitres pour l'hôtel de Ferrare à Fontainebleau ». Sur le «Grand Ferrare » envisagé d'après les documents de l'Archivio di Stato de Modène, voir C. Occhipinti, «Il "camerino" e la "galleria" nella Villa d'Este a Fontainebleau ("Hôtel de Ferrare") ", Annali della Scuola Normale Superiore di Pisa, s. IV, vol. II, 1997, p. 601-635 ; Id., « La Villa d'Este a Fontainebleau e le sue "stufette”. Documenti su Serlio e il Cardinale di Ferrara", Prospettiva, vol. 89-90, 1998, p.169-183. Sur l'imitation des modèles architecturaux mis en place par le cardinal de Ferrare, voir C. Grodecki, Documents du Minutier central des notaires de Paris. Histoire de l'art au XVI siècle (1540-1600). I, ouvr. cité, p. 111.

24. Ippolito II d'Este évoque son retour à Rome avec le nonce en 1547 et il explique pouvoir se contenter de peu - il pense au palais de Santi Apostoli - puisqu'en France «bastarian due stanzette alla francese ». La formule se révèle ambivalente puisque le cardinal signifie aussi bien le caractère rustique voire rudimentaire des logements à la cour de France que son accoutumance à ces conditions de vie. J. Lestocquoy (éd.), Correspondances des nonces en France Dandino, Della Torre et 
Trivultio (1546-1551), Paris / Rome, De Boccard / Presses de l'Université grégorienne, 1966, p. 174, Girolamo Dandino à Alessandro Farnese, Rambouillet, 29 mars 1547.

25. Sur les bénéfices d'Ippolito II d'Este, voir V. Pacifici, Ippolito II d'Este cardinale di Ferrara, Tivoli, 1920, p.331-333; J.-M. Vasseur, «1536-1550. L'irrésistible ascension d'Hippolyte le "magnifique" ", dans M. Cogotti et F. P. Fiore (éds), Ippolito II d'Este, ouvr. cité, p. 117.

26. ASMo, Carteggio ambasciatori, Francia, b. 43, copialettera, Giulio Alvarotti à Ercole II d'Este, Argentan, 11 juin 1545 : "Il Cesano legge in ogni dì, doppo desinare, il Dante a madama la Dolfina, madama Margherita e monsignor d'Orliens. Vi vanno qualche gentiluomini. Oggi, leggendo, fu un damosella che tiro, con riverenzia di Vostra Eccellenzia, una gran coregia che intorno le orechie de tutti li ascoltari di modo che ognuno dette nelle risa. Et acquetato il rumore, il predetto Cesano, che legge il Purgatorio, essendo a dichiare un passo ove si puniscono e' vizii e peccati di gola, disse: "Questo fu peccato di culo e non di gola", che reitero le risa, ma non per questo la damosella cangiò molto di colore, anzi stete intrepida come se per causa soa non si fosse fatta tutta questa pausa. " ASMo, Carteggio ambasciatori, Francia, b. 22, Giulio Alvarotti à Ercole II, Melun, 5 mai 1546 : « Si fa ora leggere dal Cesano la Castramentazione di Polibio, la quale il Cesano ha fatta mettere in disegno da un certo messer Sebastiano bolognese, il quale sta in casa di monsignor reverendissimo fratello di Vostra Eccelenzia, per consergio della sua casa di Fontanableo. »

27. A. Pugliese, « Musica e musicista alla corte di Ippolito II d'Este », dans M. Cogotti et F. P. Fiore (éds), Ippolito II d'Este, ouvr. cité, p. 479-480.

28. S. Frommel, «Ippolito II d'Este commitente in Francia: dimore e architettura dipinta », dans M. Cogotti et F. P. Fiore (éds), Ippolito II d'Este, ouvr. cité, p. 101.

29. Ibid., p. 99. Sur le portail rustique, voir aussi F. Bardati, Hommes du roi et princes de l'Église romaine. Les cardinaux français et l'art italien (1495-1560), Rome, École française de Rome, 2015, p. 85-87.

30. J.-P. Babelon, « Du grand Ferrare à Carnavalet, naissance de l'hôtel classique », Revue de l'art, vol. 41, 1978, p. 83-108.

31. C. Michon, «Hippolyte d'Este (1509-1572)», dans Id. (dir.), Les Conseillers de François I $^{\text {, }}$, Rennes, PUR, 2011, p. 528-529. L'auteur invite à ne pas exagérer le rôle politique d'Ippolito II d'Este à la cour de France. Le cardinal de Ferrare se positionne surtout en expert des affaires italiennes tout en cherchant à dessiner un espace d'autonomie politique personnel.

32. J. Lestocquoy (éd.), Correspondance des nonces en France Carpi et Ferrerio. 1535-1540, Rome / Paris, Presses de l'Université grégorienne / De Boccard, 1961, p.580, Filiberto Ferrerio à Alessandro Farnese, Sainte-Colombe, 13 juillet 1540. Voir aussi ASMo, Carteggio ambasciatori, Francia, b. 44, copialettera, Giulio Alvarotti à Ercole II d'Este Melun, 18 juillet 1546.

33. Il se positionne aussi comme compagnon des plaisirs du roi, comme le rappelle la fameuse scène de la visite aux bains, évoquée par Carlo Sacrati (ASMo, Carteggio ambasciatori, Francia, b. 16, Carlo Sacrati à Ercole II d'Este, Paris, 18 juin 1540) : « Ho inteso da Ms Thomaso del Vecchio che il giorno che S. $M^{\text {tà }}$ arrivo a Fontanableo, la sera Madama Malgherita et Madama d'Etampes con Madama di Rotolino et due altre dame erano nel bagno et $S$. $M^{\text {tà }}$ con Mons. Contestabile, et Mons ${ }^{r} R^{m o}$ di Loreno et Mons ${ }^{r}$ $R^{m o}$ Nostro, che vi anderono et le trovarano ignude, et li stettero gran pezzo a scherzare. »

34. B. Jestaz, «Les Italiens à Fontainebleau », Künstlerischer Austauch, vol. 28, 1992, p. 93-104.

35. S. Serlio, Il primo libro d'Architettura, Paris, De l'imprimerie de Jehan Barbé, 1545, fol. aaIIr.

36. B. Cellini, La Vita, L. Belloto (éd.), Liv. II, chap. IX, Parme, Fondazione Pietro Bembo - Ugo Guanda, 1996, p. 494.

37. S. Frommel, «Hippolyte d'Este à Chaalis : architecture projetée, architecture illusionniste ", Les Monuments Piot, vol. 87, 2008, p. 143-172.

38. ASMo, Carteggio ambasciatori, Francia, b. 22, Giulio Alvarotti à Ercole II, Melun, 5 mai 1546. C'est encore là qu'il réside après le retour du cardinal de Ferrare en Italie en 1549.

39. S. Frommel, Sebastiano Serlio. Architecte de la Renaissance, trad. Y. Pauwels, Paris, Gallimard, 2002, p. 28 et 219-246.

40. Ibid., p. 31-32. 
41. S. Frommel, «Sebastiano Serlio et Francesco Primaticcio. Deux architectes bolonais à la Cour de France ", dans O. Medvedkova (dir.), Les Européens. Ces architectes qui ont bâti l'Europe (1450-1950), Berne, Peter Lang, 2017, p. 41-63, aux p. 46-49.

42. ASMo, Carteggio ambasciatori, Francia, b. 20, Giulio Alvarotti à Ercole II d'Este, Melun, 29 janvier 1545.

43. B. Cellini, La Vita, Liv. II, chap. II, ouvr. cité, p. 468.

44. Le cardinal de Ferrare fait venir un artiste italien qui n'hésite pas à porter un jugement sévère sur l'art français puisqu'il parle de «lor mala maniera franciosa » (B. Cellini, La Vita, Liv. II, chap. XXI, ouvr. cité, p. 527). Voir aussi Ibid., Liv. II, chap. XXVII, p.540-543. Sur le séjour de Benvenuto Cellini à la cour de France, voir B. Jestaz, «Benvenuto Cellini et la cour de France (1540-1545) », Bibliothèque de l'École des Chartes, vol. 161, 2003, p. 71-132.

45. A. Dufour et H. Meylan (éds), Correspondance Théodore de Bèze, t. III, Genève, Librairie Droz, 1963 p. 166, Théodore de Bèze à Calvin, Saint-Germain, 27 septembre 1561.

46. P. de La Place, Commentaires de l'estat de la religion et république soubs les rois Henry et François seconds et Charles neufiesme, s. 1., 1565, fol. 235v-236v. Dans son Histoire ecclésiastique des Églises réformées, Théodore de Bèze reprend le récit de Pierre de La Place. Il se fait toutefois plus incisif en ajoutant des éléments concernant la médiocrité du cardinal et évoquant la comparaison du cardinal de Ferrare avec un renard (T. de Bèze, Histoire ecclésiastique des Églises réformées au royaume de France, t.I, G. Baum, É. Cunitz et R. Reuss (éds), Nieuwkoop, B. de Graaf, 1974, p. 615-616).

47. J. Spifame de Brou [?], Lettre adressée de Rome à la Royne Mere du Roy, traduite d'Italien en François, contenant utile admonition pour pourvoir aux affaires qui se présentent, dans Mémoires de Condé servant d'éclaircissement et de preuves à Mémoires de Condé servant d'éclaircissement et de preuves à l'Histoire de M. de Thou, t. IV, Londres / Paris, Rollin, 1743, p. 647.

48. J.-A. de Thou, Histoire universelle depuis 1543 jusqu'en 1607. Traduite de l'édition latine de Londres, Londres, s. n., 1734, p. 127-128. Voir aussi Bibliothèque nationale de France, Fr. 23110, fol. 19r. Ce passage, issu du Traité sommaire faict au sujet de la légation du cardinal Chigi venu en France en exécution du Traité de Pise 1664, rappelle les difficultés rencontrées par Ippolito II d'Este pour faire reconnaitre ses facultés par Michel de L'Hospital puis devant le Parlement de Paris. Sur cet aspect $\mathrm{du}$ gallicanisme juridique, voir A. Tallon, Conscience nationale et sentiment religieux en France au $\mathrm{XVI}^{e}$ siècle. Essai sur la vision gallicane du monde, Paris, PUF, 2002, p. 142-149.

49. H. Heller, Anti-Italianism in Sixteenth-Century France, Toronto, University of Toronto Press, 2003, p. 114-136. Parmi une importante bibliographie, on pourra aussi citer J.-F. Dubost, La France italienne, XVI ${ }^{e}-\mathrm{XVI} \mathrm{I}^{e}$ siècle, Paris, Aubier, 1997, p.307-329; sur les dimensions culturelles du phénomène, R. E. Zorach, «The Matter of Italy: Sodomy and The Scandal of Style in SixteenthCentury France », The Journal of Medieval and Early Modern Studies, vol. 28, n 3, 1998, p. 581-609. Sur les aspects littéraires de l'anti-italianisme, voir P. M. Smith, The Anti-Courtier Trend in Sixteenth Century French Literature, Genève, Librairie Droz, 1966 ; L. Sozzi, La polémique anti-italienne en France au seizième siècle, dans Id., Rome n'est plus Rome. La polémique anti-italienne et autres essais sur la Renaissance suivis de "La dignité de l'homme ", Paris, Honoré Champion, 2002, p. 9-84.

50. Voir aussi J.-F. Dubost, ouvr. cité, p. 332-335, ici p.334. Sur les rumeurs d'une SaintBarthélemy des Italiens, voir N. Le Roux, «La Saint-Barthélemy des Italiens n'aura pas lieu : un discours envoyé à Catherine de Médicis en 1573 », dans B. Barbiche, J.-P. Poussou et A. Tallon (éds), Pouvoirs, contestations et comportements dans l'Europe moderne: mélanges en l'honneur du professeur Yves-Marie Bercé, Paris, PUPS, 2005, p. 165-183.

51. M. Penzi, «L'Italia e gli italiani visti dai Francesi alla fine del XVI secolo », dans R. Russi (éd.), L'Italia vista dagli altri, Atti del I Convegno internazionale (Banka Luka, 12-13 giugno 2009), Florence, Franco Cesati editore, 2010, p. 89-94.

52. J. Balsamo, Les Rencontres des Muses. Italianisme et anti-italianisme dans les Lettres françaises de la fin $\mathrm{du} \mathrm{XVI}$ siècle, Genève, Slatkine, 1992, p. 29. 


\section{RÉSUMÉS}

Entre 1536 et 1563, Ippolito II d'Este passe quinze ans dans le royaume de France. Il joue un rôle d'intermédiaire entre l'Italie et la France, tant sur le plan politique que sur le plan culturel. Le cardinal de Ferrare, et certains Italiens de sa suite, entendent jouer sur le décalage culturel et artistique qu'ils pensent remarquer entre la cour de France et les cours italiennes. L'usage politique du mécénat artistique révèle la perception d'Ippolito II d'Este des Français, mais aussi sa conception du rôle d'un prélat issu d'une prestigieuse dynastie italienne. Or, avec les guerres de Religion, la magnificence du cardinal est tournée en dérision. Le mécénat artistique du prélat s'expose à un retournement politique dégradant son image. La vie d'Ippolito II d'Este offre ainsi la possibilité de connaître les discours d'Italiens sur les Français, et les discours que ces derniers sont en train d'élaborer sur le cardinal, au gré de leurs échanges.

Tra il 1536 e il 1563, Ippolito II d'Este trascorre quindici anni nel regno di Francia svolgendovi un ruolo politico e culturale di mediatore tra Italia e Francia. Il cardinale di Ferrara, e alcuni italiani della sua corte, intendono giocare sul divario culturale e artistico che pensano di notare tra la corte di Francia e le corti italiane. L'uso politico del patrocinio artistico rivela la percezione di Ippolito II d'Este dei Francesi, ma anche la sua concezione del ruolo di un prelato di una prestigiosa dinastia italiana. Ora, con le guerre di religione francesi, la magnificenza del cardinale viene derisa. Il patrocinio artistico del prelato è esposto a un'inversione politica che degrada la sua immagine. La vita di Ippolito II d'Este offre quindi la possibilità di conoscere i discorsi degli Italiani sui Francesi, e quelli che questi ultimi stanno elaborando sul cardinale man mano che procedono i loro scambi.

Between 1536 and 1563, Ippolito II d'Este spent fifteen years in the kingdom of France. He played the role of an intermediary between Italy and France on the political as well as the cultural level. The cardinal of Ferrara and some Italians in his retinue, intended to play on the cultural and artistic gap they thought they noticed between the French and Italian courts. The political use of artistic patronage reveals Ippolito II d'Este's perception of French people, but also his conception of the role of a prelate from a prestigious Italian dynasty. Yet, with the French Wars of Religion, the magnificence of the cardinal was mocked. The prelate's artistic patronage was exposed to a political reversal which degraded his image. Thus, Ippolito II d'Este's life provides an opportunity to know the Italian discourses on French people, and the discourses that the latter are elaborating on the Cardinal, as their exchanges unfold. 


\section{INDEX}

Mots-clés : cardinal, mécénat artistique, collectionneur, ambassadeur, érudition, humanisme, diffusion des idées et des formes, vie culturelle, réseaux d'artistes, vie artistique, histoire de la Cour, Église catholique, Renaissance, XVIe siècle, Europe, Italie, Rome, France

Keywords : cardinal, artistic patronage, collector, ambassador, erudition, humanism, dissemination of ideas and forms, cultural life, artists' networks, artistic life, Court history, Catholic Church, Renaissance, 16th century, Europe, Italy, Rome, France

Parole chiave : cardinale, mecenatismo artistico, collezionista, ambasciatore, erudizione, umanesimo, diffusione di idee e forme, vita culturale, reti di artisti, vita artistica, storia della Corte, Chiesa cattolica, Rinascimento, Cinquecento, Europa, Italia, Roma, Francia

\section{AUTEUR}

\section{JEAN SÉNIÉ}

Sorbonne Université, Centre Roland Mousnier (UMR 8596)

jean.senie@gmail.com 